\title{
Correction to: Secondary prevention of osteoporotic fractures: evaluation of the Lille University Hospital's Fracture Liaison Service between January 2016 and January 2018
}

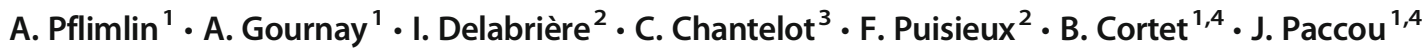 \\ Published online: 8 July 2019 \\ (C) International Osteoporosis Foundation and National Osteoporosis Foundation 2019
}

\section{Correction to: Osteoporosis International} https://doi.org/10.1007/s00198-019-05036-0

In the original version of this article, published on 5 June 2019, an author's name was misspelled.

The correct author name reads as follows:

A. Pflimlin

The original article has been corrected.

The online version of the original article can be found at https://doi.org/10. 1007/s00198-019-05036-0

J. Paccou

julien.paccou@chru-lille.fr

A. Pflimlin

Arnaud.PFLIMLIN@CHRU-LILLE.FR

A. Gournay

Alexandre.GOURNAY@CHRU-LILLE.FR

I. Delabrière

Isabelle.DELABRIERE@CHRU-LILLE.FR

C. Chantelot

Christophe.CHANTELOT@CHRU-LILLE.FR
F. Puisieux

Francois.PUISIEUX@CHRU-LILLE.FR

B. Cortet

Bernard.CORTET@CHRU-LILLE.FR

Department of Rheumatology, Lille University Hospital, Lille, France

2 Department of Gerontology, Lille University Hospital, Lille, France

3 Department of Traumatology, Lille University Hospital, Lille, France

4 PMOI, Lille University-ULCO, EA 4490, 59000 Lille, France 